\title{
An Existence Result for Quasilinear Parabolic Systems with Lower Order Terms
}

\section{Farah Balaadich and Elhoussine Azroul}

Faculty of Sciences Dhar El Mehraz, University of Sidi Mohamed Ben

Abdellah

B.P. 1796 Atlas, Fez-Morocco

E-mail(corresp.): balaadich.edp@gmail.com

E-mail: elhoussine.azroul@gmail.com

Received September 14, 2020; revised October 14, 2021; accepted October 14, 2021

Abstract. In this paper we prove the existence of weak solutions for a class of quasilinear parabolic systems, which correspond to diffusion problems, in the form

$$
\left\{\begin{array}{rlr}
\frac{\partial u}{\partial t}-\operatorname{div} \sigma(x, t, u, D u) & =v(x, t)+f(x, t, u)+\operatorname{div} g(x, t, u) \quad \text { in } \Omega \times(0, T), \\
u(x, t) & =0 \text { on } \partial \Omega \times(0, T), \\
u(x, 0) & =u_{0}(x) \text { in } \Omega,
\end{array}\right.
$$

where $\Omega$ is a bounded open domain of $\mathbb{R}^{n}, 0<T<\infty$ be given and $u_{0} \in L^{2}\left(\Omega ; \mathbb{R}^{m}\right)$. The function $v$ belongs to $L^{p^{\prime}}\left(0, T: W^{-1, p^{\prime}}\left(\Omega ; \mathbb{R}^{m}\right)\right)$ is in a moving and dissolving substance, the dissolution is described by $f$ and the motion by $g$. We prove the existence result by using Galerkin's approximation and the theory of Young measures. Keywords: quasilinear parabolic systems, weak solutions, Young measures.

AMS Subject Classification: 35K55; 35D30.

\section{Introduction}

Let $\Omega$ be a bounded open set of $\mathbb{R}^{n}, n \geq 2, T$ is a positive real number, $Q=\Omega \times(0, T)$ and $1<p<\infty$. In this paper, we consider the following quasilinear parabolic system:

$$
\begin{aligned}
\frac{\partial u}{\partial t}-\operatorname{div} \sigma(x, t, u, D u) & =v(x, t)+f(x, t, u)+\operatorname{div} g(x, t, u) \quad \text { in } Q, \\
u(x, t) & =0 \quad \text { on } \partial \Omega \times(0, T), \\
u(x, 0) & =u_{0}(x) \quad \text { in } \Omega,
\end{aligned}
$$

Copyright (C) 2021 The Author(s). Published by Vilnius Gediminas Technical University This is an Open Access article distributed under the terms of the Creative Commons Attribution License (http://creativecommons.org/licenses/by/4.0/), which permits unrestricted use, distribution, and reproduction in any medium, provided the original author and source are credited. 
where $u: Q \rightarrow \mathbb{R}^{m}$ is a vector-valued function and $D u$ its gradient which belongs to $\mathbb{M}^{m \times n}$. Here $\mathbb{M}^{m \times n}$ stands for the real vector space of $m \times n$ matrices equipped with the inner product $\xi: \eta=\sum_{i=1}^{m} \sum_{j=1}^{n} \xi_{i j} \eta_{i j}$. The functions $\sigma: Q \times \mathbb{R}^{m} \times \mathbb{M}^{m \times n} \rightarrow \mathbb{M}^{m \times n}, f: Q \times \mathbb{R}^{m} \rightarrow \mathbb{R}^{m}$ and $g: Q \times \mathbb{R}^{m} \rightarrow \mathbb{M}^{m \times n}$ are assumed to satisfy some conditions (see below). Moreover, the function $v: Q \rightarrow$ $\mathbb{R}^{m}$ is in $L^{p^{\prime}}\left(0, T ; W^{-1, p^{\prime}}\left(\Omega ; \mathbb{R}^{m}\right)\right)$ the dual space of $L^{p}\left(0, T: W_{0}^{1, p}\left(\Omega ; \mathbb{R}^{m}\right)\right)$, with $p^{\prime}=p /(p-1)$ the conjugate exponent of $p$.

Consider first the quasilinear elliptic system

$$
-\operatorname{div} \sigma(x, u, D u)=f \quad \text { in } \Omega,
$$

endowed with the Dirichlet boundary condition. The existence result is proved in [14] by Hungerbühler. The author used the tool of Young measures and weak monotonicity over $\sigma$ to achieve his result. See also [2] for a generalized $p$-Laplacian system. We find a generalization of (1.4) in [1], where the following quasilinear elliptic system

$$
-\operatorname{div} \sigma(x, u, D u)=v(x)+f(x, u)+\operatorname{div} g(x, u) \quad \text { in } \Omega
$$

was considered. This system corresponds to a diffusion problem with a source $v$ in a moving and dissolving substance, where the motion is described by $g$ and the dissolution by $f$. The authors proved existence of a weak solution for this system under classical regularity, growth, and coercivity conditions for $\sigma$, but with only very mild monotonicity assumptions. See also $[3,6]$ for more results.

For the evolutionary problems, Hungerbühler [15] considered

$$
\frac{\partial u}{\partial t}-\operatorname{div} \sigma(x, t, u, D u)=v \quad \text { in } Q
$$

with the initial and boundary conditions (1.2)-(1.3), where $v \in L^{p^{\prime}}(0, T$ : $\left.W^{-1, p^{\prime}}\left(\Omega ; \mathbb{R}^{m}\right)\right)$ for some $p \in\left(\frac{2 n}{n+2}, \infty\right)$ and $u_{0} \in L^{2}\left(\Omega ; \mathbb{R}^{m}\right)$. The existence of a weak solution under classical regularity, growth, and coercivity conditions for $\sigma$ but with only very mild monotonicity assumptions is proved. We have extended in [4] the problem (1.6) to a more general strongly quasilinear parabolic system containing the lower term $g(x, t, u, D u)$ in the following form

$$
\frac{\partial u}{\partial t}-\operatorname{div} \sigma(x, t, u, D u)+g(x, t, u, D u)=v \quad \text { in } Q .
$$

Under mild monotonicity assumptions on $\sigma$, we have proved the existence of weak solutions. The elliptic case of (1.7) can be found in [5], where we have investigated another mild monotonicity condition, called the strict quasimonotone. Note that, in all works mentioned above, the authors used the theory of Young measures to achieve their results, since the classical monotone operator theory can not be used for some reasons (see Remark 1). See also $[8,9,10,18,19]$ for similar problems.

Inspired by the previous works (especially $[4,15]$ ), we want to study the existence result for the problem (1.1)-(1.3). This work, can be seen as an extension of [1] (i.e. of (1.5)) to a parabolic case and generalizes both works 
$[4,15]$. We will use the Young measure as a technical tool to obtain the desired result.

The paper is organized as follows: In Section 2, we specify the assumptions on $\sigma, f, g$ and $u_{0}$ needed in the present study and introduce the definition of a weak solution of (1.1)-(1.3). We present in Section 3 an overview on Young measures, while Section 4 is devoted to present the main result and its proof.

\section{Assumptions on the data and the definition of a weak solution}

Throughout this paper, we suppose that the following assumptions hold true: $\Omega$ is a bounded open set of $\mathbb{R}^{n}(n \geq 2), T>0$ is given and we set $Q=\Omega \times(0, T)$. Moreover, we assume:

(H0)(Continuity) $\sigma: Q \times \mathbb{R}^{m} \times \mathbb{M}^{m \times n} \rightarrow \mathbb{M}^{m \times n}$ is a Carathéodory function, i.e. measurable w.r.t $(x, t) \in Q$ and continuous w.r.t other variables.

(H1)(Growth and coercivity) There exist $\alpha_{1} \geq 0, \alpha_{2}>0, d_{1} \in L^{p^{\prime}}(Q)$ and $d_{2} \in L^{1}(Q)$ such that

$$
\begin{aligned}
& |\sigma(x, t, s, \xi)| \leq d_{1}(x, t)+\alpha_{1}\left(|s|^{p-1}+|\xi|^{p-1}\right), \\
& \sigma(x, t, s, \xi): \xi \geq \alpha_{2}|\xi|^{p}-d_{2}(x, t)
\end{aligned}
$$

for almost every $(x, t) \in Q$ and all $(s, \xi) \in \mathbb{R}^{m} \times \mathbb{M}^{m \times n}$.

(H2)(Monotonicity) $\sigma$ satisfies one of the following conditions:

(a) For a.e. $(x, t) \in Q$ and all $s \in \mathbb{R}^{m}, \xi \mapsto \sigma(x, t, s, \xi)$ is a $C^{1}$-function and is monotone, i.e.,

$$
(\sigma(x, t, s, \xi)-\sigma(x, t, s, \eta)):(\xi-\eta) \geq 0
$$

for all $\xi, \eta \in \mathbb{M}^{m \times n}$.

(b) There exists a function (potential) $W: Q \times \mathbb{R}^{m} \times \mathbb{M}^{m \times n} \rightarrow \mathbb{R}$ such that $\sigma(x, t, s, \xi)=D_{\xi} W(x, t, s, \xi):=\frac{\partial W}{\partial \xi}(x, t, s, \xi)$, and $\xi \mapsto W(x, t, s, \xi)$ is convex and $C^{1}$ for a.e. $(x, t) \in Q$ and all $s \in \mathbb{R}^{m}$.

(c) $\sigma$ is strictly monotone, i.e., $\sigma$ is monotone and

$$
(\sigma(x, t, s, \xi)-\sigma(x, t, s, \eta)):(\xi-\eta)=0 \quad \text { implies } \quad \xi=\eta \text {. }
$$

(d) $\sigma$ is strictly $p$-quasimonotone, i.e.,

$$
\int_{\mathbb{M}^{m \times n}}(\sigma(x, t, s, \lambda)-\sigma(x, t, s, \bar{\lambda})):(\lambda-\bar{\lambda}) d \nu_{(x, t)}(\lambda)>0,
$$

where $\bar{\lambda}=\left\langle\nu_{(x, t)}, i d\right\rangle$ and $\nu=\left\{\nu_{(x, t)}\right\}_{(x, t) \in Q}$ is any family of Young measures generated by a bounded sequence in $L^{p}(Q)$ and not a Dirac measure for a.e. $(x, t) \in Q$.

(H3)(i)(Continuity) $f: Q \times \mathbb{R}^{m} \rightarrow \mathbb{R}^{m}$ is a Carathéodory function in the sense of $(\mathrm{H} 0)$.

(ii)(Growth) There exist $\alpha_{3} \geq 0,0<\gamma<p-1$ and $d_{3} \in L^{p^{\prime}}(Q)$ such that

$$
|f(x, t, s)| \leq d_{3}(x, t)+\alpha_{3}|s|^{\gamma},
$$


for a.e. $(x, t) \in Q$ and all $s \in \mathbb{R}^{m}$.

(H4)(i)(Continuity) $g: Q \times \mathbb{R}^{m} \rightarrow \mathbb{M}^{m \times n}$ is a Carathéodory function in the sense of $(\mathrm{H} 0)$.

(ii)(Growth) There exist $\alpha_{4} \geq 0,0<\rho<p-1$ and $d_{4} \in L^{p^{\prime}}(Q)$ such that

$$
|g(x, t, s)| \leq d_{4}(x, t)+\alpha_{4}|s|^{\rho},
$$

for a.e. $(x, t) \in Q$ and all $s \in \mathbb{R}^{m}$.

Remark 1. Assumption (H2)(b) allows to take a potential $W(x, t, s, \xi)$, which is only convex but not strictly convex in $\xi \in \mathbb{M}^{m \times n}$, and to consider (1.1) with $\sigma=D_{\xi} W$. Note that if $W$ is assumed to be strictly convex, then $\sigma$ becomes strictly monotone and the standard monotone operator may apply, but it is not the case in this paper.

A prototype of our problem (1.1)-(1.3) can be given by

$$
\left\{\begin{aligned}
\frac{\partial u}{\partial t}-\operatorname{div}\left(|D u|^{p-2} D u\right) & =v(x, t)+|u|^{\gamma}+\operatorname{div}\left(a(x, t)|u|^{\rho}\right) \quad \text { in } Q \\
u(x, t) & =0 \text { on } \partial \Omega \times(0, T) \\
u(x, 0) & =u_{0}(x) \text { in } \Omega
\end{aligned}\right.
$$

for $0<\gamma<p-1,0<\rho<p-1$ and $a: Q \rightarrow \mathbb{M}^{m \times n}$ is a measurable function and bounded. For the potential $W$, one can take $W:=\frac{1}{p}|\xi|^{p}$ for $\xi \in \mathbb{M}^{m \times n}$.

Now, we can define the weak solution of (1.1)-(1.3) as follows:

DeFinition 1. A weak solution of (1.1)-(1.3) is a function

$$
u \in L^{p}\left(0, T ; W_{0}^{1, p}\left(\Omega ; \mathbb{R}^{m}\right)\right) \cap L^{\infty}\left(0, T ; L^{2}\left(\Omega ; \mathbb{R}^{m}\right)\right)
$$

such that

$$
\begin{aligned}
& \int_{0}^{T}\left\langle\frac{\partial u}{\partial t}, \varphi\right\rangle d t+\int_{Q} \sigma(x, t, u, D u): D \varphi \\
& \quad=\int_{0}^{T}\langle v, \varphi\rangle d t+\int_{Q} f(x, t, u) \cdot \varphi d x d t-\int_{Q} g(x, t, u): D \varphi d x d t
\end{aligned}
$$

holds for every $\varphi \in L^{p}\left(0, T ; W_{0}^{1, p}\left(\Omega ; \mathbb{R}^{m}\right)\right)$. Here $\langle.,$.$\rangle denotes the dual pairing$ of $W^{-1, p^{\prime}}\left(\Omega ; \mathbb{R}^{m}\right)$ and $W_{0}^{1, p}\left(\Omega ; \mathbb{R}^{m}\right)$.

\section{A review on Young measures}

As stated in the introduction, we use the tool of Young measures to prove the existence result. This concept of Young measures is a nice tool to understand and control difficulties that arises when weak convergence does not behave as one desires with respect to nonlinear functionals and operators. For convenience of the readers not familiar with this concept, we give an overview needed in this paper. See $[7,12,13]$ for more details.

By $C_{0}\left(\mathbb{R}^{m}\right)$ we denote the Banach space of continuous functions on $\mathbb{R}^{m}$ which satisfies $\lim _{|\lambda| \rightarrow \infty} \varphi(\lambda)=0$. Its dual is the well known space of signed 
Radon measures with finite mass denoted as $\mathcal{M}\left(\mathbb{R}^{m}\right)$. The related duality is given for $\nu: \Omega \rightarrow \mathcal{M}\left(\mathbb{R}^{m}\right)$, by

$$
\langle\nu, \varphi\rangle=\int_{\mathbb{R}^{m}} \varphi(\lambda) d \nu(\lambda) .
$$

A particular case of $\varphi$ is the identity $i d$, thus $\langle\nu, i d\rangle=\int_{\mathbb{R}^{m}} \lambda d \nu(\lambda)$.

Lemma 1. [12] Let $\left\{z_{j}\right\}_{j \geq 1}$ be a measurable sequence in $L^{\infty}\left(\Omega ; \mathbb{R}^{m}\right)$. Then there exists a subsequence $\left\{z_{k}\right\}_{k} \subset\left\{z_{j}\right\}_{j}$ and a Borel probability measure $\nu_{x}$ on $\mathbb{R}^{m}$ for almost every $x \in \Omega$, such that for $\varphi \in C_{0}\left(\mathbb{R}^{m}\right)$ we have

$$
\varphi\left(z_{k}\right) \rightarrow^{*} \bar{\varphi} \text { weakly in } L^{\infty}\left(\Omega ; \mathbb{R}^{m}\right),
$$

where $\bar{\varphi}(x)=\left\langle\nu_{x}, \varphi\right\rangle=\int_{\mathbb{R}^{m}} \varphi(\lambda) d \nu_{x}(\lambda)$ for almost every $x \in \Omega$.

Definition 2. The map $\nu_{x}: \Omega \rightarrow \mathcal{M}\left(\mathbb{R}^{m}\right)$ in Lemma 1 is called the Young measure generated by the subsequence $\left\{z_{k}\right\}_{k}$.

Lemma 2. [13] (1) If $|\Omega|<\infty$ and $\nu_{x}$ is the Young measure generated by the (whole) sequence $z_{j}$, then there holds

$$
z_{j} \rightarrow z \quad \text { in measure } \Longleftrightarrow \nu_{x}=\delta_{z(x)} \quad \text { for a.e. } x \in \Omega .
$$

(2) If further $w_{j}: \Omega \rightarrow \mathbb{R}^{d}$ generates the Young measure $\delta_{w(x)}$, then $\left(z_{j}, w_{j}\right)$ generates the Young measure $\nu_{x} \otimes \delta_{w(x)}$.

Remark 2. (1) It is shown in [7] that for any Carathéodory function $\varphi: \Omega \times$ $\mathbb{R}^{m} \rightarrow \mathbb{R}$ and $\left\{z_{k}\right\}_{k}$ generating a Young measure $\nu_{x}$, we have

$$
\varphi\left(x, z_{k}\right) \rightarrow^{*}\left\langle\nu_{x}, \varphi(x, .)\right\rangle=\int_{\mathbb{R}^{m}} \varphi(x, \lambda) d \nu_{x}(\lambda)
$$

weakly in $L^{1}\left(\Omega^{\prime}\right)$ for all measurable $\Omega^{\prime} \subset \Omega$, provided that the negative part $\varphi^{-}\left(x, z_{k}\right)$ is equiintegrable.

(2) The previous properties remain true on $\mathbb{M}^{m \times n}$ if we replace $z_{k}$ by $D w_{k}$, where $w_{k}: \Omega \rightarrow \mathbb{R}^{m}$.

Lemma 3. [4] If $\left\{D w_{k}\right\}_{k}$ is bounded in $L^{p}\left(Q ; \mathbb{M}^{m \times n}\right)$, then the Young measure $\nu_{(x, t)}$ generated by D $w_{k}$ has the following properties:

(i) $\nu_{(x, t)}$ is a probability measure, i.e., $\left\|\nu_{(x, t)}\right\|_{\mathcal{M}\left(\mathbb{M}^{m \times n}\right)}:=\int_{\mathbb{M}^{m \times n}} d \nu_{(x, t)}(\lambda)=1$ for almost every $(x, t) \in Q$.

(ii) The weak $L^{1}$-limit of D $w_{k}$ is given by $\left\langle\nu_{(x, t)}, i d\right\rangle=\int_{\mathbb{M}^{m \times n}} \lambda d \nu_{(x, t)}(\lambda)$.

(iii) $\nu_{(x, t)}$ satisfies $\left\langle\nu_{(x, t)}, i d\right\rangle=D u(x, t)$ for almost every $(x, t) \in Q$.

We conclude this section by recalling the following useful Fatou-type inequality.

Lemma 4. [11] Let $\varphi: Q \times \mathbb{R}^{m} \times \mathbb{M}^{m \times n} \rightarrow \mathbb{R}$ be a Carathéodory function and $w_{k}: Q \rightarrow \mathbb{R}^{m}$ a sequence of measurable functions such that $w_{k} \rightarrow w$ in measure and such that D $w_{k}$ generates the Young measure $\nu_{(x, t)}$, with $\left\|\nu_{(x, t)}\right\|_{\mathcal{M}}=1$ for almost every $(x, t) \in Q$. Then

$$
\liminf _{k \rightarrow \infty} \int_{Q} \varphi\left(x, t, w_{k}, D w_{k}\right) d x d t \geq \int_{Q} \int_{\mathbb{M}^{m \times n}} \varphi(x, t, w, \lambda) d \nu_{x}(\lambda) d x d t
$$

provided that the negative part $\varphi^{-}\left(x, t, w_{k}, D w_{k}\right)$ is equiintegrable. 


\section{Existence result}

In this section we present the main result and its proof. Consider the quasilinear parabolic system (1.1)-(1.3). Let $p$ be a real number such that $1<p<\infty$. The result of this paper reads as follows:

Theorem 1. If $\sigma$ satisfies the conditions (H0)-(H2), then the problem (1.1)(1.3) has a weak solution $u \in L^{p}\left(0, T ; W_{0}^{1, p}\left(\Omega ; \mathbb{R}^{m}\right)\right) \cap C\left(0, T ; L^{2}\left(\Omega ; \mathbb{R}^{m}\right)\right)$ for every $v \in L^{p^{\prime}}\left(0, T ; W^{-1, p^{\prime}}\left(\Omega ; \mathbb{R}^{m}\right)\right)$, every $f$ satisfying (H3) and every $g$ satisfying $\left(\mathrm{H}_{4}\right)$.

Proof. The proof is divided into five steps. In Step 1, we present local approximating solutions by the well known Galerkin method. Step 2 is devoted to extend these solutions to the whole interval $[0, T]$. In Step 3, some a priori estimates will be presented. Step 4 shows the div-curl inequality which is the key ingredient to pass to the limit in Step 5.

Step 1: We define

$$
u_{k}(x, t)=\sum_{i=1}^{k} \alpha_{k i}(t) w_{i}(x)
$$

to approximate the solutions of (1.1)-(1.3), where $\alpha_{k i}:[0, T) \rightarrow \mathbb{R}^{+}$satisfy

$$
\begin{aligned}
& \int_{\Omega} \frac{\partial u_{k}}{\partial t} w_{i}(x) d x+\int_{\Omega} \sigma\left(x, t, u_{k}, D u_{k}\right): D w_{i}(x) d x \\
& =\left\langle v(t), w_{i}\right\rangle+\int_{\Omega} f\left(x, t, u_{k}\right) \cdot w_{i}(x) d x-\int_{\Omega} g\left(x, t, u_{k}\right): D w_{i}(x) d x
\end{aligned}
$$

and $\left\{w_{i}\right\}_{i \geq 1}$ is an $L^{2}$-orthonormal base, such that

$$
\left\{w_{i}\right\}_{i \geq 1} \subset C_{0}^{\infty}\left(\Omega ; \mathbb{R}^{m}\right) \subset \overline{k \geq 1}_{V_{k}}^{C^{1}\left(\bar{\Omega} ; \mathbb{R}^{m}\right)},
$$

where $V_{k}=\operatorname{span}\left\{w_{1}, . ., w_{k}\right\}$. Assume that $u_{k} \in L^{p}\left(0, T ; W_{0}^{1, p}\left(\Omega ; \mathbb{R}^{m}\right)\right)$, thus $u_{k}$ satisfies the condition (1.2) by construction. For the condition (1.3), we can choose $\alpha_{k i}(0):=\left(u_{0}, w_{i}\right)_{L^{2}(\Omega)}$ such that

$$
u_{k}(., 0)=\sum_{i=1}^{k} \alpha_{k i}(0) w_{i}(.) \longrightarrow u_{0} \quad \text { in } L^{2}(\Omega) \quad \text { as } k \rightarrow \infty
$$

where $(., .)_{L^{2}}$ is the inner product of $L^{2}\left(\Omega ; \mathbb{R}^{m}\right)$. Let us fix $k \in \mathbb{N}, 0<\tau<T$ and set $I=[0, \tau]$. We choose $r>0$ large enough, such that $B_{r}(0):=B(0, r) \subset$ $\mathbb{R}^{k}$ contains the vectors $\left(\alpha_{k 1}(0), . ., \alpha_{k k}(0)\right)$. Consider the operator

$$
\begin{aligned}
T: I \times \overline{B_{r}(0)} & \longrightarrow \mathbb{R}^{k} \\
\left(t, \alpha_{1}, . ., \alpha_{k}\right) \mapsto\left(\left\langle v(t), w_{j}\right\rangle\right. & +\int_{\Omega} f\left(x, t, u_{k}\right) \cdot w_{j} d x-\int_{\Omega} g\left(x, t, u_{k}\right): D w_{j} d x \\
& \left.\quad-\int_{\Omega} \sigma\left(x, t, u_{k}, D u_{k}\right): D w_{j} d x\right)_{j=1, \ldots, k},
\end{aligned}
$$


where $u_{k}:=\sum_{i=1}^{k} \alpha_{k i}(t) w_{i}(x) . T$ is a Carathéodory function by (H0), (H3)(i) and (H4)(i). By the growth conditions in (H1), (H3)(ii) and (H4)(ii), we can write (without loss of generality, we can assume that $\gamma=p-1$ and $\rho=p-1$ )

$$
\begin{aligned}
& \int_{\Omega}\left|\sigma\left(x, t, u_{k}, D u_{k}\right)\right|^{p^{\prime}} d x \leq c \int_{\Omega}\left(\left|d_{1}(x, t)\right|^{p^{\prime}}+\left|u_{k}\right|^{p}+\left|D u_{k}\right|^{p}\right) d x \\
& \int_{\Omega}\left|f\left(x, t, u_{k}\right)\right|^{p^{\prime}} d x \leq c \int_{\Omega}\left(\left|d_{3}(x, t)\right|^{p^{\prime}}+\left|u_{k}\right|^{p}\right) d x \\
& \int_{\Omega}\left|g\left(x, t, u_{k}\right)\right|^{p^{\prime}} d x \leq c \int_{\Omega}\left(\left|d_{4}(x, t)\right|^{p^{\prime}}+\left|u_{k}\right|^{p}\right) d x
\end{aligned}
$$

for a positive constant $c$ which may change values from line to line. By Hölder's inequality, it follows for the component $T_{j}$ that it can be estimated as follows $\left|T_{j}\left(t, \alpha_{1}, . ., \alpha_{k}\right)\right| \leq C(r, k) l(t)$ uniformly on $I \times \overline{B_{r}(0)}$, where $l(t) \in L^{1}(I)$ does not depend on $r$ and $k$, and $C(r, k)$ is a constant which depends on $k$ and $r$. Therefore, the Carathéodory existence result on ordinary differential equations (see, e.g. Kamke [16]) applied to the system

$$
\left\{\begin{aligned}
\alpha_{j}^{\prime}(t) & =T_{j}\left(t, \alpha_{1}(t), \ldots, \alpha_{k}(t)\right) \\
\alpha_{j}(0) & =\alpha_{k j}(0)
\end{aligned}\right.
$$

for $j=1, \ldots, k$ ensures existence of a distributional, continuous solution $\alpha_{j}$ depending on $k$ of the Equation (4.2) on a time interval $\left[0, \tau^{\prime}\right)$, where $\tau^{\prime}>0, a$ priori may depend on $k$. The corresponding integral equation of (4.2) is given by

$$
\alpha_{j}(t)=\alpha_{j}(0)+\int_{0}^{t} T_{j}\left(s, \alpha_{1}(s), \ldots, \alpha_{k}(s)\right) d s
$$

holds on $\left[0, \tau^{\prime}\right)$. Hence $u_{k}(x, t)=\sum_{i=1}^{k} \alpha_{k i}(t) w_{i}(x)$ is the desired solution of (4.1) with the initial condition $u_{k}(., 0)=\sum_{i=1}^{k} \alpha_{k i}(0) w_{i}(.) \rightarrow u_{0}$ in $L^{2}\left(\Omega ; \mathbb{R}^{m}\right)$ for $k \rightarrow \infty$.

Step 2: Now, we extend the local solution constructed in Step 1 to the whole interval $[0, T)$. To this purpose, we multiply the Equation (4.1) by $\alpha_{k j}(t)$ and we sum over $j=1, \ldots, k$, we get for $\tau \in[0, T)$

$$
\begin{aligned}
& \int_{Q_{\tau}} \frac{\partial u_{k}}{\partial t} u_{k} d x d t+\int_{Q_{\tau}} \sigma\left(x, t, u_{k}, D u_{k}\right): D u_{k} d x d t \\
& =\int_{0}^{\tau}\left\langle v(t), u_{k}\right\rangle d t+\int_{Q_{\tau}} f\left(x, t, u_{k}\right) \cdot u_{k} d x d t-\int_{Q_{\tau}} g\left(x, t, u_{k}\right): D u_{k} d x d t
\end{aligned}
$$

where $Q_{\tau}=\Omega \times(0, \tau)$. We have

$$
I_{1} \equiv \int_{Q_{\tau}} \frac{\partial u_{k}}{\partial t} u_{k} d x d t=\frac{1}{2}\left\|u_{k}(., \tau)\right\|_{L^{2}(\Omega)}^{2}-\frac{1}{2}\left\|u_{k}(., 0)\right\|_{L^{2}(\Omega)}^{2} .
$$

The coercivity condition in (H1) yields

$I_{2} \equiv \int_{Q_{\tau}} \sigma\left(x, t, u_{k}, D u_{k}\right): D u_{k} d x d t \geq \alpha_{2} \int_{Q_{\tau}}\left|D u_{k}\right|^{p} d x d t-\int_{Q_{\tau}} d_{2}(x, t) d x d t$. 
By Hölder's inequality and the growth condition (H3)(i) and (H4)(i), it follows that

$$
\begin{aligned}
&\left|I_{3}\right| \equiv\left|\int_{0}^{\tau}\left\langle v(t), u_{k}\right\rangle d t\right| \leq\|v\|_{-1, p^{\prime}}\left\|u_{k}\right\|_{1, p}, \\
&\left|I_{4}\right| \equiv\left|\int_{Q_{\tau}} f\left(x, t, u_{k}\right) \cdot u_{k} d x d t\right| \leq\left\|d_{3}\right\|_{p^{\prime}}\left\|u_{k}\right\|_{p}+\alpha_{3}\left\|u_{k}\right\|_{p}^{\gamma+1} \\
& \leq \theta\left\|d_{3}\right\|_{p^{\prime}}\left\|D u_{k}\right\|_{p}+\alpha_{3} \theta^{\gamma+1}\left\|D u_{k}\right\|_{p}^{\gamma+1}, \\
&\left|I_{5}\right| \equiv\left|\int_{Q_{\tau}} g\left(x, t, u_{k}\right): D u_{k} d x d t\right| \leq\left\|d_{4}\right\|_{p^{\prime}}\left\|D u_{k}\right\|_{p}+\alpha_{4}\left\|u_{k}\right\|_{p}^{\rho}\left\|D u_{k}\right\|_{p} \\
& \leq\left\|d_{4}\right\|_{p^{\prime}}\left\|D u_{k}\right\|_{p}+\alpha_{4} \theta^{\rho}\left\|D u_{k}\right\|_{p}^{\rho+1},
\end{aligned}
$$

where $\theta$ is the constant of Poincaré's inequality:

$$
\|z\|_{p} \leq \theta\|D z\|_{p} \quad \forall z \in L^{p}\left(0, T ; W_{0}^{1, p}\left(\Omega ; \mathbb{R}^{m}\right)\right) .
$$

The constant $\theta$ depends on $\operatorname{diam}(\Omega)$ (see e.g. [17]). We know that $u_{k}(x, 0)=$ $\psi_{k}(x) \longrightarrow u_{0}$ in $L^{2}(\Omega)$, thus

$$
\int_{\Omega} u_{k}^{2}(x, 0) d x=\int_{\Omega}\left|\psi_{k}(x)\right|^{2} d x \leq c \quad \text { for all } k .
$$

Consequently, from the estimates on $I_{\epsilon}, \epsilon=1, \ldots, 5$ we deduce that $\left\|u_{k}(., \tau)\right\|_{L^{2}(\Omega)}^{2} \leq c$. Let us consider now

$$
\Lambda:=\{t \in[0, T): \text { there exists a weak solution of }(4.2) \text { on }[0, t)\} .
$$

The set $\Lambda$ is non-empty since it contains a local solution. Moreover, it is an open set and closed (see e.g. [15]). Therefore, $\Lambda=[0, T)$.

Step 3: By virtue of the estimations on $I_{\epsilon}, \epsilon=1, \ldots, 5$, we can write

$$
\begin{aligned}
& \frac{1}{2}\left\|u_{k}(., \tau)\right\|_{L^{2}(\Omega)}^{2}+\alpha_{2} \int_{Q_{\tau}}\left|D u_{k}\right|^{p} d x d t \leq \frac{1}{2}\left\|u_{k}(., 0)\right\|_{L^{2}(\Omega)}^{2}+\left\|d_{2}\right\|_{L^{1}\left(Q_{\tau}\right)} \\
& \quad+\theta\left\|d_{3}\right\|_{p^{\prime}}\left\|D u_{k}\right\|_{p}+\|v\|_{-1, p^{\prime}}\left\|u_{k}\right\|_{1, p} \\
& \quad+\alpha_{3} \theta^{\gamma+1}\left\|D u_{k}\right\|_{p}^{\gamma+1}+\left\|d_{4}\right\|_{p^{\prime}}\left\|D u_{k}\right\|_{p}+\alpha_{4} \theta^{\rho}\left\|D u_{k}\right\|_{p}^{\rho+1} .
\end{aligned}
$$

If $\left\|D u_{k}\right\|_{p}$ is unbounded, then $\int_{Q_{\tau}}\left|D u_{k}\right|^{p} d x d t$ is unbounded, and this contra$\operatorname{dict}(4.3)$ since $p>\max \{1, \gamma+1, \rho+1\}$. Therefore the sequence $\left(u_{k}\right)$ is bounded in $L^{p}\left(0, T ; W_{0}^{1, p}\left(\Omega ; \mathbb{R}^{m}\right)\right) \cap L^{\infty}\left(0, T ; L^{2}\left(\Omega ; \mathbb{R}^{m}\right)\right)$. For a suitable subsequence (still denoted by $\left(u_{k}\right)$ ),

$$
u_{k} \rightarrow u \text { in } L^{p}\left(0, T ; W_{0}^{1, p}\left(\Omega ; \mathbb{R}^{m}\right)\right), \quad u_{k} \rightarrow^{*} u \text { in } L^{\infty}\left(0, T ; L^{2}\left(\Omega ; \mathbb{R}^{m}\right)\right) .
$$

The function $u \in L^{p}\left(0, T ; W_{0}^{1, p}\left(\Omega ; \mathbb{R}^{m}\right)\right) \cap L^{\infty}\left(0, T ; L^{2}\left(\Omega ; \mathbb{R}^{m}\right)\right)$ is a candidate to be a weak solution for (1.1)-(1.3).

Owing to the growth conditions (H1), (H3)(ii) and (H4)(ii), we obtain

$$
\begin{aligned}
& \int_{Q}\left|\sigma\left(x, t, u_{k}, D u_{k}\right)\right|^{p^{\prime}} d x d t \leq c \int_{Q}\left(\left|d_{1}(x, t)\right|^{p^{\prime}}+c\left(\left|u_{k}\right|^{p}+\left|D u_{k}\right|^{p}\right)\right) d x d t \\
& \int_{Q}\left|f\left(x, t, u_{k}\right)\right|^{p^{\prime}} d x d t \leq c \int_{Q}\left(\left|d_{3}(x, t)\right|^{p^{\prime}}+c\left|u_{k}\right|^{p}\right) d x d t
\end{aligned}
$$


and

$$
\int_{Q}\left|g\left(x, t, u_{k}\right)\right|^{p^{\prime}} d x d t \leq c \int_{Q}\left(\left|d_{4}(x, t)\right|^{p^{\prime}}+c\left|u_{k}\right|^{p}\right) d x d t
$$

(without loss of generality, we have assumed $\gamma=p-1$ and $\rho=p-1$ ). Since $d_{1}, d_{3}, d_{4} \in L^{p^{\prime}}(Q)$ and $\left(u_{k}\right)_{k}$ is bounded in $L^{p}\left(0, T ; W_{0}^{1, p}\left(\Omega ; \mathbb{R}^{m}\right)\right)$, then

$$
\sigma\left(x, t, u_{k}, D u_{k}\right) \rightarrow \chi, \quad f\left(x, t, u_{k}\right) \rightarrow F, \quad g\left(x, t, u_{k}\right) \rightarrow G
$$

in $L^{p^{\prime}}(Q)$ (for a proper subsequence). According to the argument of AubinSimon (see also [4]) we then have $u \in C\left(0, T ; L^{2}\left(\Omega ; \mathbb{R}^{m}\right)\right)$ and there is a weak convergence $u_{k}(., T) \rightarrow u(., T)$ in $L^{2}(\Omega)$.

Step 4: From Step 3, we have that $\left(u_{k}\right)_{k}$ is bounded in $L^{p}\left(0, T ; W_{0}^{1, p}\left(\Omega ; \mathbb{R}^{m}\right)\right)$. Then by Lemma 1, it follows the existence of a Young measure $\nu_{(x, t)}$ generated by $D u_{k}$ in $L^{p}(Q)$ such that $\nu_{(x, t)}$ satisfies the properties of Lemma 3. Now, we show the following lemma, namely a div-curl inequality, which is the key ingredient to pass to the limit in the approximating equations.

Lemma 5. Suppose that $\sigma, f$ and $g$ satisfy (HO)-(H4). Then the Young measure $\nu_{(x, t)}$ associated to Du $u_{k}$ has the following property:

$$
\int_{Q} \int_{\mathbb{M}^{m \times n}}(\sigma(x, t, u, \lambda)-\sigma(x, t, u, D u)):(\lambda-D u) d \nu_{(x, t)}(\lambda) d x d t \leq 0 .
$$

Proof. Let consider the sequence

$$
\begin{aligned}
I_{k} & :=\left(\sigma\left(x, t, u_{k}, D u_{k}\right)-\sigma(x, t, u, D u)\right):\left(D u_{k}-D u\right) \\
& =\sigma\left(x, t, u_{k}, D u_{k}\right):\left(D u_{k}-D u\right)-\sigma(x, t, u, D u):\left(D u_{k}-D u\right) \\
& =: I_{k, 1}+I_{k, 2} .
\end{aligned}
$$

As in the Equation (4.4), $\sigma(., u, D u) \in L^{p^{\prime}}\left(Q ; \mathbb{M}^{m \times n}\right)$ for arbitrary $u \in$ $L^{p}\left(0, T ; W_{0}^{1, p}\left(\Omega ; \mathbb{R}^{m}\right)\right)$. Then by virtue of Lemma 3 , we can write

$$
\begin{gathered}
\liminf _{k \rightarrow \infty} \int_{Q} I_{k, 2} d x d t=\int_{Q} \int_{\mathbb{M}^{m \times n}} \sigma(x, t, u, D u):(\lambda-D u) d \nu_{(x, t)}(\lambda) d x d t \\
=\int_{Q} \sigma(x, t, u, D u):(\underbrace{\int_{\mathbb{M}^{m \times n}} \lambda d \nu_{(x, t)}(\lambda)}_{=: D u(x, t)}-D u) d x d t=0 .
\end{gathered}
$$

Since $u_{k} \rightarrow u$ in $L^{p}\left(0, T ; W_{0}^{1, p}\left(\Omega ; \mathbb{R}^{m}\right)\right)$, then $u_{k} \rightarrow u$ in measure (for a subsequence). By the growth condition in (H1), $\left(\sigma\left(x, t, u_{k}, D u_{k}\right): D u\right)^{-}$is equiintegrable. Now, let $Q^{\prime} \subset Q$ be measurable. The coercivity condition in (H1) yields

$$
\begin{aligned}
\int_{Q^{\prime}} \mid \min ( & \left.\sigma\left(x, t, u_{k}, D u_{k}\right): D u_{k}, 0\right) \mid d x d t \\
& \leq \alpha_{2} \int_{Q^{\prime}}\left|D u_{k}\right|^{p} d x d t+\int_{Q^{\prime}}\left|d_{2}(x, t)\right| d x d t<\infty
\end{aligned}
$$


Hence $\left(\sigma\left(x, t, u_{k}, D u_{k}\right): D u_{k}\right)^{-}$is equiintegrable. According to Lemma 4 , it follows that

$$
I:=\liminf _{k \rightarrow \infty} \int_{Q} I_{k} d x d t \geq \int_{Q} \int_{\mathbb{M}^{m \times n}} \sigma(x, t, u, \lambda):(\lambda-D u) d \nu_{(x, t)}(\lambda) d x d t .
$$

It is now sufficient to show that $I \leq 0$. By Step 3, we have the following energy equality which is the first property of $\chi, F$ and $G$ :

$$
\begin{aligned}
\frac{1}{2}\|u(., T)\|_{L^{2}(\Omega)}^{2} & -\frac{1}{2}\|u(., 0)\|_{L^{2}(\Omega)}^{2}+\int_{Q} \chi: D u d x d t \\
& =\int_{0}^{T}\langle v(t), u\rangle d t+\int_{Q} F \cdot u d x d t-\int_{Q} G: D u d x d t .
\end{aligned}
$$

On the one hand, we have

$$
\begin{aligned}
& \liminf _{k \rightarrow \infty}\left(-\int_{Q} \sigma\left(x, t, u_{k}, D u_{k}\right): D u d x d t\right)=-\int_{Q} \chi: D u d x d t \\
= & \frac{1}{2}\|u(., T)\|_{L^{2}(\Omega)}^{2}-\frac{1}{2}\|u(., 0)\|_{L^{2}(\Omega)}^{2}-\int_{0}^{T}\langle v(t), u\rangle d t-\int_{Q} F \cdot u d x d t \\
& +\int_{Q} G: D u d x d t .
\end{aligned}
$$

On the other hand, the Galerkin equations allow to write

$$
\begin{gathered}
\liminf _{k \rightarrow \infty} \int_{Q} \sigma\left(x, t, u_{k}, D u_{k}\right): D u_{k} d x d t=\liminf _{k \rightarrow \infty}\left(-\int_{Q} \frac{\partial u_{k}}{\partial t} u_{k} d x d t\right. \\
\left.+\int_{0}^{T}\left\langle v(t), u_{k}\right\rangle d t+\int_{Q} f\left(x, t, u_{k}\right) \cdot u_{k} d x d t-\int_{Q} g\left(x, t, u_{k}\right): D u_{k} d x d t\right) \\
\leq-\frac{1}{2}\|u(., T)\|_{L^{2}(\Omega)}^{2}+\frac{1}{2}\|u(., 0)\|_{L^{2}(\Omega)}^{2}+\int_{0}^{T}\langle v(t), u\rangle d t \\
+\int_{Q} F \cdot u d x d t-\int_{Q} G: D u d x d t
\end{gathered}
$$

where we have used $u_{k}(., T) \rightarrow u(., T)$ in $L^{2}\left(\Omega ; \mathbb{R}^{m}\right)$ and $u_{k}(., 0) \rightarrow u_{0}()=$. $u(., 0)$ as $k \rightarrow \infty$. The combination of (4.7) and (4.8) implies

$$
I=\liminf _{k \rightarrow \infty} \int_{Q} \sigma\left(x, t, u_{k}, D u_{k}\right):\left(D u_{k}-D u\right) d x d t \leq 0
$$

as desired.

Step 5: In this step, we will pass to the limit in the Galerkin equations by considering the conditions $(a)-(d)$ listed in $(\mathrm{H} 2)$. Note that, as in [4], we have

$$
(\sigma(x, t, u, \lambda)-\sigma(x, t, u, D u)):(\lambda-D u)=0 \quad \text { on } \operatorname{supp} \nu_{(x, t)} .
$$

Let start with the case $(c)$ : the strict monotonicity of $\sigma$ together with (4.9) implies that $\nu_{(x, t)}=\delta_{D u(x, t)}$. By virtue of Lemma 2, it follows that $D u_{k} \rightarrow D u$ 
in measure and almost everywhere in $Q$ as $k \rightarrow \infty$. The continuity of $\sigma$ gives $\sigma\left(x, t, u_{k}, D u_{k}\right) \rightarrow \sigma(x, t, u, D u)$ almost everywhere. Since $\sigma\left(x, t, u_{k}, D u_{k}\right)$ is bounded (see the Equation (4.4)), then $\sigma\left(x, t, u_{k}, D u_{k}\right) \rightarrow \sigma(x, t, u, D u)$ in $L^{\beta}(Q), \forall \beta \in\left[0, p^{\prime}\right)$ by the Vitali convergence theorem. Hence

$$
\sigma\left(x, t, u_{k}, D u_{k}\right) \rightarrow \chi=\sigma(x, t, u, D u) \quad \text { in } L^{p^{\prime}}(Q) .
$$

For the case (d), we suppose by contradiction that $\nu_{(x, t)}$ is not a Dirac measure on a set $(x, t) \in Q^{\prime} \subset Q$ of positive Lebesgue measure. We have by the strict $p$-quasimonotone of $\sigma$ that

$$
\begin{aligned}
0 & <\int_{Q} \int_{\mathbb{M}^{m \times n}}(\sigma(x, t, u, \lambda)-\sigma(x, t, u, \bar{\lambda})):(\lambda-\bar{\lambda}) d \nu_{(x, t)}(\lambda) d x d t \\
& =\int_{Q} \int_{\mathbb{M}^{m \times n}} \sigma(x, t, u, \lambda):(\lambda-\bar{\lambda}) d \nu_{(x, t)}(\lambda) d x d t,
\end{aligned}
$$

where we have used

$$
\begin{aligned}
\int_{Q} \int_{\mathbb{M}^{m \times n}} \sigma(x, t, u, \bar{\lambda}) & :(\lambda-\bar{\lambda}) d \nu_{(x, t)}(\lambda) d x d t \\
=\int_{Q} \sigma(x, t, u, \bar{\lambda}): & (\underbrace{\int_{\mathbb{M}^{m \times n}} \lambda d \nu_{(x, t)}(\lambda)}_{=: \bar{\lambda}}-\bar{\lambda}) d x d t=0 .
\end{aligned}
$$

Thus

$$
\int_{Q} \int_{\mathbb{M}^{m} m n} \sigma(x, t, u, \lambda): \lambda d \nu_{(x, t)}(\lambda) d x d t>\int_{Q} \int_{\mathbb{M}^{m \times n}} \sigma(x, t, u, \lambda): \bar{\lambda} d \nu_{(x, t)}(\lambda) d x d t .
$$

By virtue of Lemma 5 (i.e. $I \leq 0$ ), we then have

$$
\begin{gathered}
\int_{Q} \int_{\mathbb{M}^{m \times n}} \sigma(x, t, u, \lambda): \bar{\lambda} d \nu_{(x, t)}(\lambda) d x d t \geq \int_{Q} \int_{\mathbb{M}^{m \times n}} \sigma(x, t, u, \lambda): \lambda d \nu_{(x, t)}(\lambda) d x d t \\
>\int_{Q} \int_{\mathbb{M}^{m \times n}} \sigma(x, t, u, \lambda): \bar{\lambda} d \nu_{(x, t)}(\lambda) d x d t
\end{gathered}
$$

which is a contradiction. Therefore $\nu_{(x, t)}=\delta_{h(x, t)}$. Then

$$
h(x, t)=\int_{\mathbb{M}^{m \times n}} \lambda d \delta_{h(x, t)}(\lambda)=\int_{\mathbb{M}^{m \times n}} \lambda d \nu_{(x, t)}(\lambda)=D u(x, t) .
$$

Hence $\nu_{(x, t)}=\delta_{D u(x, t)}$ and by virtue of Lemma 2, Du $u_{k} \rightarrow D u$ in measure for $k \rightarrow \infty$. The remains of the proof in this case is similar to that in case $(c)$.

Consider now the case $(a)$. Let us prove first that for all $\xi \in \mathbb{M}^{m \times n}$

$$
\sigma(x, t, u, \lambda): \xi=\sigma(x, t, u, D u): \xi+(\nabla \sigma(x, t, u, D u) \xi):(D u-\lambda)
$$

holds on $\operatorname{supp} \nu_{(x, t)}$, where $\nabla$ is the derivative of $\sigma$ with respect to its last variable.

The monotonicity of $\sigma$ allows to write for all $\tau \in \mathbb{R}$ and $\xi \in \mathbb{M}^{m \times n}$

$$
(\sigma(x, t, u, \lambda)-\sigma(x, t, u, D u+\tau \xi)):(\lambda-D u-\tau \xi) \geq 0,
$$


thus

$$
\begin{aligned}
& -\sigma(x, t, u, \lambda): \tau \xi \geq-\sigma(x, t, u, \lambda):(\lambda-D u) \\
& \quad+\sigma(x, t, u, D u+\tau \xi):(\lambda-D u-\tau \xi) \\
& \quad=-\sigma(x, t, u, D u):(\lambda-D u)+\sigma(x, t, u, D u+\tau \xi):(\lambda-D u-\tau \xi)
\end{aligned}
$$

by (4.9). Using the fact

$$
\sigma(x, t, u, D u+\tau \xi)=\sigma(x, t, u, D u)+\nabla \sigma(x, t, u, D u) \tau \xi+o(\tau),
$$

we deduce that

$$
-\sigma(x, t, u, \lambda): \tau \xi \geq \tau[(\nabla \sigma(x, t, u, D u) \xi):(\lambda-D u)-\sigma(x, t, u, D u): \xi]+o(\tau) .
$$

Since $\tau$ is arbitrary in $\mathbb{R}$, the needed equality (4.10) follows. As $\sigma\left(x, t, u_{k}, D u_{k}\right)$ is equiintegrable, then its weak $L^{1}$-limit is given by

$$
\begin{aligned}
\bar{\sigma}:= & \int_{\mathbb{M}^{m \times n}} \sigma(x, t, u, \lambda) d \nu_{(x, t)}(\lambda)=\int_{\operatorname{supp} \nu_{(x, t)}} \sigma(x, t, u, \lambda) d \nu_{(x, t)}(\lambda) \stackrel{(4.10)}{=} \int_{\operatorname{supp} \nu_{(x, t)}}(\sigma(x, t, u, D u) \\
& +(\nabla \sigma(x, t, u, D u)):(D u-\lambda)) d \nu_{(x, t)}(\lambda)=\sigma(x, t, u, D u) \int_{\operatorname{supp} \nu_{(x, t)}} d \nu_{(x, t)}(\lambda) \\
& +(\nabla \sigma(x, t, u, D u))^{t}: \int_{\operatorname{supp} \nu_{(x, t)}}(D u-\lambda) d \nu_{(x, t)}(\lambda)=\sigma(x, t, u, D u) .
\end{aligned}
$$

Consequently

$$
\sigma\left(x, t, u_{k}, D u_{k}\right) \rightarrow \sigma(x, t, u, D u) \quad \text { in } L^{p^{\prime}}(Q) .
$$

For the case (b), we show that $\operatorname{supp} \nu_{(x, t)} \subset K_{(x, t)}$, where

$$
K_{(x, t)}=\left\{\lambda \in \mathbb{M}^{m \times n}: W(x, t, u, \lambda)=W(x, t, u, D u)+\sigma(x, t, u, D u):(\lambda-D u)\right\} .
$$

Let $\lambda \in K_{(x, t)}$, then by the Equation (4.9)

$$
(1-\tau)(\sigma(x, t, u, \lambda)-\sigma(x, t, u, D u)):(\lambda-D u)=0 \quad \forall \tau \in[0,1] .
$$

Using this equation and the monotonicity of $\sigma$ to obtain

$$
\begin{aligned}
0 & \leq(1-\tau)(\sigma(x, t, u, D u+\tau(\lambda-D u))-\sigma(x, t, u, \lambda)):(D u-\lambda) \\
& =(1-\tau)(\sigma(x, t, u, D u+\tau(\lambda-D u))-\sigma(x, t, u, D u)):(D u-\lambda) .
\end{aligned}
$$

Using again the monotonicity of $\sigma$ for the right hand side of the above inequality, yields

$$
(\sigma(x, t, u, D u+\tau(\lambda-D u))-\sigma(x, t, u, D u)): \tau(\lambda-D u) \geq 0,
$$

which implies since $\tau \in[0,1]$ that

$$
(\sigma(x, t, u, D u+\tau(\lambda-D u))-\sigma(x, t, u, D u)):(1-\tau)(\lambda-D u) \geq 0 .
$$


From (4.11) and (4.12) it follows that

$$
(\sigma(x, t, u, D u+\tau(\lambda-D u))-\sigma(x, t, u, D u)):(\lambda-D u)=0 \quad \forall \tau \in[0,1],
$$

i.e.,

$$
\sigma(x, t, u, D u+\tau(\lambda-D u)):(\lambda-D u)=\sigma(x, t, u, D u):(\lambda-D u),
$$

whenever $\lambda \in \operatorname{supp} \nu_{(x, t)}$. Integrate the Equation (4.13) over [0,1] and use the fact that $\sigma:=D_{\xi} W$, we deduce that

$$
\begin{aligned}
W(x, t, u, \lambda) & =W(x, t, u, D u)+\int_{0}^{1} \sigma(x, t, u, D u+\tau(\lambda-D u)):(\lambda-D u) d \tau \\
& =W(x, t, u, D u)+\sigma(x, t, u, D u):(\lambda-D u)
\end{aligned}
$$

Therefore $\operatorname{supp} \nu_{(x, t)} \subset K_{(x, t)}$. The convexity of $W$ implies for all $\lambda \in \mathbb{M}^{m \times n}$

$$
\underbrace{W(x, t, u, \lambda)}_{=: A(\lambda)} \geq \underbrace{W(x, t, u, D u)+\sigma(x, t, u, D u):(\lambda-D u)}_{=: B(\lambda)} .
$$

Since $\lambda \mapsto A(\lambda)$ is a $C^{1}$-function, then for $\xi \in \mathbb{M}^{m \times n}$ and $\tau \in \mathbb{R}$ we have

$$
\begin{array}{ll}
\frac{A(\lambda+\tau \xi)-A(\lambda)}{\tau} \geq \frac{B(\lambda+\tau \xi)-B(\lambda)}{\tau} & \text { for } \tau>0 \\
\frac{A(\lambda+\tau \xi)-A(\lambda)}{\tau} \leq \frac{B(\lambda+\tau \xi)-B(\lambda)}{\tau} & \text { for } \tau<0 .
\end{array}
$$

Thus $D_{\lambda} A=D_{\lambda} B$, i.e.,

$$
\sigma(x, t, u, \lambda)=\sigma(x, t, u, D u) \quad \forall \lambda \in K_{(x, t)} \supset \operatorname{supp} \nu_{(x, t)},
$$

and then

$$
\begin{aligned}
\bar{\sigma} & =\int_{\mathbb{M}^{m \times n}} \sigma(x, t, u, \lambda) d \nu_{(x, t)}(\lambda)=\int_{\operatorname{supp} \nu_{(x, t)}} \sigma(x, t, u, \lambda) d \nu_{(x, t)}(\lambda) \\
& =\int_{\operatorname{supp} \nu_{(x, t)}} \sigma(x, t, u, D u) d \nu_{(x, t)}(\lambda)=\sigma(x, t, u, D u) .
\end{aligned}
$$

Consider the Carathéodory function $h(x, t, s, \lambda)=|\sigma(x, t, s, \lambda)-\bar{\sigma}(x, t)|$. The equiintegrability of $\sigma\left(x, t, u_{k}, D u_{k}\right)$ implies that $h_{k}(x, t):=h\left(x, t, u_{k}, D u_{k}\right)$ is equiintegrable, and its weak $L^{1}$-limit is given as

$$
\begin{aligned}
\bar{h}(x, t) & =\int_{\mathbb{R}^{m} \times \mathbb{M}^{m \times n}} h(x, t, s, \lambda) d \delta_{u(x, t)}(s) \otimes d \nu_{(x, t)}(\lambda) \\
& =\int_{\operatorname{supp} \nu_{(x, t)}}|\sigma(x, t, u, \lambda)-\bar{\sigma}(x, t)| d \nu_{(x, t)}(\lambda)=0 \quad(\text { by }(4.14),(4.15)) .
\end{aligned}
$$

The weak $L^{1}$-limit of $h_{k}$ is in fact strong since $h_{k} \geq 0$. Therefore

$$
h_{k} \longrightarrow 0 \quad \text { in } L^{1}(Q) \text {. }
$$


Since $h_{k}$ is bounded in $L^{p^{\prime}}(Q)$, the Vitali convergence theorem implies that $\sigma\left(x, t, u_{k}, D u_{k}\right) \rightarrow \sigma(x, t, u, D u)$ in $L^{p^{\prime}}(Q)$.

To conclude the proof of Theorem 1 , it remains to pass to the limit on $f\left(x, t, u_{k}\right)$ and $g\left(x, t, u_{k}\right)$. Since $u_{k} \rightarrow u$ in measure for $k \rightarrow \infty$, we may infer that, after extraction of a suitable subsequence, if necessary,

$$
u_{k} \rightarrow u \quad \text { almost everywhere for } k \rightarrow \infty \text {. }
$$

Thus for arbitrary $\varphi \in L^{p}\left(0, T ; W_{0}^{1, p}\left(\Omega ; \mathbb{R}^{m}\right)\right)$, it follows from the continuity property in (H3)(i) and (H4) (i) that $f\left(x, t, u_{k}\right) \cdot \varphi \rightarrow f(x, t, u) \cdot \varphi$ and $g\left(x, t, u_{k}\right)$ : $D \varphi \rightarrow g(x, t, u): D \varphi$ almost everywhere. Since, by (4.5) and (4.6), $f\left(x, t, u_{k}\right)$ and $g\left(x, t, u_{k}\right)$ are equiintegrable, it follows that $f\left(x, t, u_{k}\right) \cdot \varphi \rightarrow f(x, t, u) \cdot \varphi$ and $g\left(x, t, u_{k}\right): D \varphi \rightarrow g(x, t, u): D \varphi$ in $L^{1}(Q)$ by the Vitali convergence theorem.

Now, we take a test function $w \in \cup_{i \in \mathbb{N}} V_{i}$ and $\phi \in C_{0}^{\infty}([0, T])$ in (4.1) and integrate over $(0, T)$ and pass to the limit $k \rightarrow \infty$. The resulting equation is

$$
\begin{aligned}
& \int_{Q} \frac{\partial u}{\partial t} \phi(t) w(x) d x d t+\int_{Q} \sigma(x, t, u, D u): D w(x) \phi(t) d x d t \\
& =\int_{0}^{T}\langle v(t), \phi w\rangle d t+\int_{Q} f(x, t, u) \cdot \phi(t) w(x) d x d t-\int_{Q} g(x, t, u): D w(x) \phi(t) d x d t
\end{aligned}
$$

for arbitrary $w \in \cup_{i \in \mathbb{N}} V_{i}$ and $\phi \in C_{0}^{\infty}([0, T])$. By density of the linear span of these functions in $L^{p}\left(0, T ; W_{0}^{1, p}\left(\Omega ; \mathbb{R}^{m}\right)\right)$, this proves that $u$ is in fact a weak solution. Hence the proof of Theorem 1 is complete.

\section{References}

[1] F. Augsburger and N. Hungerbühler. Quasilinear elliptic systems in divergence form with weak monotonicity and nonlinear physical data. Electronic Journal of Differential Equations, 144:1-18, 2004.

[2] E. Azroul and F. Balaadich. Weak solutions for generalized p-Laplacian systems via Young measures. Moroccan J. of Pure and Appl. Anal. (MJPAA), 4(2):77-84, 2018. https://doi.org/10.1515/mjpaa-2018-0008.

[3] E. Azroul and F. Balaadich. Quasilinear elliptic systems in perturbed form. Int. J. Nonlinear Anal. Appl, 10(2):255-266, 2019.

[4] E. Azroul and F. Balaadich. Strongly quasilinear parabolic systems in divergence form with weak monotonicity. Khayyam J. Math., 6(1):57-72, 2020.

[5] E. Azroul and F. Balaadich. On strongly quasilinear elliptic systems with weak monotonicity. J. Appl. Anal., 27(1):153-162, 2021. https://doi.org/10.1515/jaa2020-2041.

[6] E. Azroul and F. Balaadich. A weak solution to quasilinear elliptic problems with perturbed gradient. Rend. Circ. Mat. Palermo II, 70:151-166, 2021. https://doi.org/10.1007/s12215-020-00488-4.

[7] J.M. Ball. A version of the fundamental theorem for Young measures. In M. Rascle, D. Serre and M. Slemrod(Eds.), PDEs and Continuum Models of Phase Transitions, volume 344, pp. 207-215, Berlin, Heidelberg, 1989. Springer Berlin Heidelberg. https://doi.org/10.1007/BFb0024945. 
[8] V. Bögelein, F. Duzaar, L. Schätzler and C. Scheven. Existence for evolutionary problems with linear growth by stability methods. J. Differential Equations, 266(11):7709-7748, 2019. https://doi.org/10.1016/j.jde.2018.12.012.

[9] M. Caddick and E. Suli. Numerical approximation of Youngmeasure solutions to parabolic systems of forward-backward type. Applicable Analysis and Discrete Mathematics, 13(3):649-696, 2019. https://doi.org/10.2298/AADM190325026C.

[10] S. Demoulini. Young measure solutions for a nonlinear parabolic equation of forward-backward type. SIAM Journal on Mathematical Analysis, 27(2):376403, 1994. https://doi.org/10.1137/S0036141094261847.

[11] G. Dolzmann, N. Hungerbühler and S. Müller. Nonlinear elliptic systems with measure-valued right hand side. Mathematische zeitschrift, 226:545-574, 1997. https://doi.org/10.1007/PL00004354.

[12] L.C. Evans. Weak convergence methods for nonlinear partial differential equations. CBMS Reg. Conf. Ser. in Math., 74, 1990. https://doi.org/10.1090/cbms/074.

[13] N. Hungerbühler. A refinement of Ball's theorem on Young measures. New York J. Math, 3:48-53, 1997.

[14] N. Hungerbühler. Quasilinear elliptic systems in divergence form with weak monotonicity. New York J. Math., 5:83-90, 1999.

[15] N. Hungerbühler. Quasilinear parabolic systems in divergence form with weak monotonicity. Duke mathematical journal, 107(3):497-519, 2001. https://doi.org/10.1215/S0012-7094-01-10733-3.

[16] E. Kamke. Das Lebesgue-Stieltjes-Integral. Teubner, Leipzig, 1960.

[17] G.M. Lieberman. The natural generalizationj of the natural conditions of Ladyzhenskaya and Ural'tseva for elliptic equations. Communications in Partial Differential Equations, 16(2-3):311-361, 1991. https://doi.org/10.1080/03605309108820761.

[18] P. Marcellini. A variational approach to parabolic equations under general and p, q-growth conditions. Nonlinear Analysis, 194:111-456, 2020. https://doi.org/10.1016/j.na.2019.02.010.

[19] R. Di Nardo, F. Feo and O. Guibe. Existence result for nonlinear parabolic equations with lower order terms. Anal. Appl.(Singap.), 9(2):161-186, 2011. https://doi.org/10.1142/S0219530511001790. 